\title{
Diffusion-Perfusion Mismatch in Symptomatic Vasospasm After Subarachnoid Hemorrhage
}

\author{
Tatsuya OHTONARI, Kenichi KAKINUMA, Tomohiro KITO, \\ Isamu EZUKA, and Tsutomu KANAZAWA* \\ Departments of Neurosurgery and *Radiology, Cerebrovascular Center, \\ Niigata Rosai Hospital, Joetsu, Niigata
}

\begin{abstract}
Diffusion-weighted and perfusion-weighted magnetic resonance (MR) imaging were investigated as a method to detect diffusion-perfusion mismatch in the early stages of vasospasm in 17 patients with acute subarachnoid hemorrhage after aneurysm clipping. Single photon emission computed tomography (SPECT) with N-isopropyl-p-[123 $\left.{ }^{12}\right]$ iodoamphetamine was also performed. Diffusion-perfusion mismatch was clearly identified in the 3 patients who manifested clinical deterioration. Perfusion-weighted imaging showed increased mean transit time, normal cerebral blood flow, and increased or normal cerebral blood volume. SPECT revealed no earlier signs of vasospasm. Diffusion-perfusion mismatch was clearly demonstrated in the early stages of vasospasm, so may be useful for early identification of ischemia in vasospasm and initiating appropriate treatment.
\end{abstract}

Key words: diffusion-perfusion mismatch, vasospasm, subarachnoid hemorrhage, single photon emission computed tomography

\section{Introduction}

Vasospasm often causes irreversible cerebral ischemia after subarachnoid hemorrhage (SAH), but is not easy to identify for early intervention. Angiography, transcranial Doppler sonography, single photon emission computed tomography (SPECT), and xenon computed tomography (CT) have all been assessed for earlier vasospasm detection. However, angiography or transcranial Doppler sonography are not suitable for identifying symptomatic vasospasm because they give no direct information about tissue perfusion, whereas SPECT or xenon CT are not developed enough for early detection of reversible ischemia. ${ }^{1,6,8-21,25-27,30)}$ Diffusion-perfusion mismatch has been identified in the ultra-acute stage of cerebral ischemia, ${ }^{2-5,11,28)}$ and may be useful for determining the indications for thrombolysis in the acute stage of cerebral infarction..$^{727,29,30)}$ We previously tried to predict vasospasm using a magnetic resonance (MR) imaging time-intensity curve on the basis of perfusion-weighted imaging, diffusionweighted imaging, and SPECT taken on the same day.

The present study tried to evaluate the cerebral circulation during vasospasm in detail by detecting diffusion-perfusion mismatch in patients with symptomatic vasospasm after SAH.

\section{Methods}

\section{Patient selection}

Seventeen patients, 5 males and 12 females (mean age $63.2 \pm 6.1$ years), were admitted to our institution with SAH after aneurysm rupture. Hunt and Kosnik grades on admission were as follows: grade 1 4 cases, grade 23 cases, grade 35 cases, and grade 4 5 cases. The interval from onset to clipping surgery was $1.0 \pm 0.1$ days. The interval from onset to examination was $7.2 \pm 0.6$ days. CT indicated no serious problems after surgery. If neurological deterioration such as hemiparesis, somnolence, or aphasia became apparent, intensive therapy involving induced hypertension, intracisternal administration of papaverine hydrochloride, and administration of red cell concentrate with mannitol-adenine-

Received August 6, 2007; Accepted May 7, 2008

Author's present address: Tatsuya Ohtonari, M.D., Department of Neurosurgery, Brain Attack Center Ota Memorial Hospital, Fukuyama, Hiroshima, Japan. 
phosphate were performed. Perfusion-weighted MR imaging and SPECT were performed on the same day to compare the measurements of cerebral blood flow (CBF). Patients gave informed consent to participation in the study.

\section{MR imaging protocol}

MR imaging was performed with a Magnetom Vision 1.5-T MRI unit (Siemens AG, Erlangen, Germany) using single shot echo-planar imaging, spin echo type diffusion-weighted imaging, and gradient echo type perfusion-weighted imaging. We used the same acute stroke MR imaging protocol described below throughout this study, which requires the patient to remain within the magnet for 30 minutes (about 15 minutes scan time).

Echo-planar images are more sensitive to susceptibility artifacts, but the nonferromagnetic clips used caused minimal artifacts on axial images above the circle of Willis. Aneurysm clip artifacts were localized near the clip. Isotropic diffusion-weighted and time to peak (TTP) images were processed automatically at the MR console and were typically available within 5 minutes after imaging was completed, and in many cases just after the protocol had finished. Mean transit time (MTT), CBF, and cerebral blood volume (CBV) images involved additional postprocessing requiring 30 minutes.

\section{Diffusion-weighted MR imaging}

The high-b value was $1000 \mathrm{sec} / \mathrm{mm}^{2}$, and the low-b value was $0 \mathrm{sec} / \mathrm{mm}^{2}$, with parameters as follows: repetition time $3900 \mathrm{msec}$, echo time $100 \mathrm{msec}$, matrix $128 \times 128$, fields of view $240 \mathrm{~cm}^{2}$, slice thickness $5 \mathrm{~mm}$, and interslice gap $5 \mathrm{~mm}$. The complete 7 image acquisition required 31 seconds, so 2 repetitions were typically acquired to improve the signalto-noise ratio. Generated isotropic diffusion-weighted images were transferred from the MR console to the work-station (Dr. View/LINUX R 2.0; Asahi Kasei Information Systems Corporation, Tokyo) by digital imaging and communication in medicine.

\section{Perfusion-weighted imaging}

Gradient echo echo-planar imaging was obtained after intravenous injection of $0.2 \mathrm{mmol} / \mathrm{kg}$ of meglumine gadopentetate by hand over 4 seconds. A total of 65 single-shot echo-planar images (repetition time $1000 \mathrm{msec}$, echo time $60.7 \mathrm{msec}$ ) in each of 7 slices for a total of 455 complete images were acquired in 65 seconds. Data were transferred to work-stations for further analysis. In our protocol, the perfusion slices were supposed to be identical to the diffusion slices. Factors identifying cerebral circulation from intravascular tracers were determined by the decon- volution method.

\section{SPECT protocol}

CBF imaging was performed with a ring type SPECT scanner (Headtome SET-080; Shimadzu Co., Kyoto) using $222 \mathrm{MBq}$ of N-isopropyl-p-[ $\left.{ }^{123} I\right]$ iodoamphetamine (123I-IMP). The scanner simultaneously produces 32 tomographic axial images covering the whole brain. A low energy, high resolution collimator was used for data acquisition with image matrix size of $128 \times 128$. A Butterworth filter with a cutoff frequency of 0.25 cycles/pixel and a ramp filter were used for image reconstruction. The planar and axial spatial resolutions were 14 and $22 \mathrm{~mm}$ full width at half maximum, respectively. CBF imaging was initiated 20 minutes after the injection of ${ }^{123}$ I-IMP, and data acquisition continued for 20 minutes. The image slices were parallel to the orbitomeatal line with a $5 \mathrm{~mm}$ interslice distance. Reconstruction images were corrected for tissue absorption using an attenuation coefficient of $0.1 / \mathrm{cm}$.

\section{Results}

Fourteen of the 17 patients suffered no apparent symptomatic vasospasm manifesting as somnolent state, hemiparesis, or aphasia during their clinical course. A 55-year-old female in Hunt and Kosnik grade 2 on admission is a typical example. The interval from onset to clipping surgery was 2 days, and from onset to examination was 9 days. Perfusionweighted imaging showed normal CBF (Fig. 1A), MTT (Fig. 1B), and CBV (Fig. 1C). Diffusion-weighted imaging showed no high intensity area except for slight, diffuse high intensity signals indicating $\mathrm{SAH}$ in the right sylvian fissure (Fig. 1D). SPECT also showed no apparent changes in the brain circulation (Fig. 1E). $\mathrm{T}_{2}$-weighted MR imaging taken 3 months after surgery revealed no apparent abnormality (Fig. $1 F)$.

The other 3 patients developed somnolent state, hemiparesis, or aphasia, and diffusion/perfusionweighted imaging and SPECT clearly demonstrated diffusion-perfusion mismatch as illustrated in Figs. 2-4, and summarized in Table 1. In Case 1, perfusion-weighted imaging showed only normal CBF (Fig. 2A), but increased MTT (Fig. 2B) and CBV (Fig. 2C) extending widely to the right hemisphere. Diffusion-weighted imaging showed no high intensity area except for very small high intensity signals of hematoma in the right sylvian fissure and the anterior horn of the lateral ventricle (Fig. 2D). In Case 2 with an old cerebral infarction in the right parietal lobe, perfusion-weighted imaging showed normal CBF (Fig. 3A), but increased MTT (Fig. 3B) and CBV 


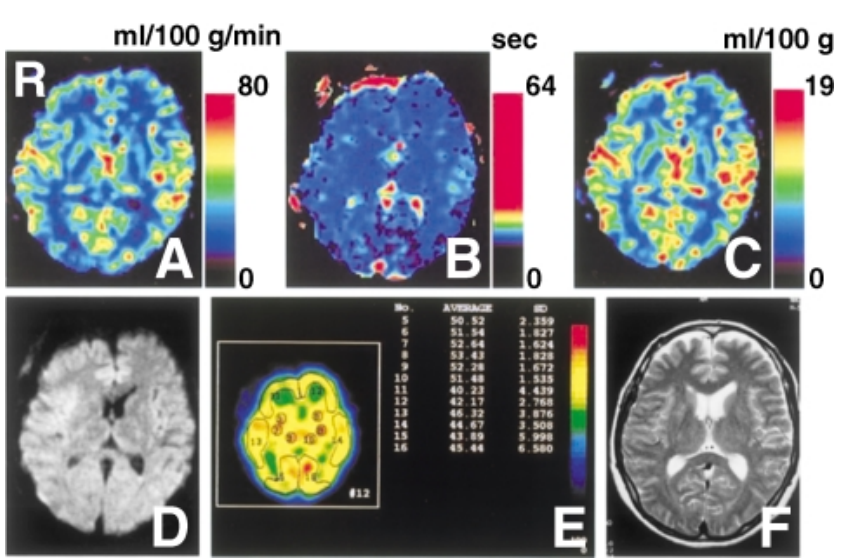

Fig. 1 Representative case without diffusion-perfusion mismatch. A-C: Perfusion-weighted magnetic resonance (MR) images showing normal cerebral blood flow (A), mean transit time (B), and cerebral blood volume (C). D: Diffusion-weighted MR image showing no high intensity area except for slight and diffuse high intensity signals indicating subarachnoid hemorrhage in the right sylvian fissure. E: Single photon emission computed tomography scan showing no apparent changes in the brain circulation. $F: T_{2}$-weighted $M R$ image taken 3 months after surgery revealing no apparent abnormality.

(Fig. 3C) in the right frontal lobe. Diffusion-weighted imaging showed no high intensity area except for some small high intensity signals of hematoma in the bilateral temporoparietal cerebral sulci and a small infarction in the white matter anterior to the left lateral ventricle (Fig. 3D). In Case 3, perfusionweighted imaging showed normal CBF (Fig. 4A) and CBV (Fig. 4C), but increased MTT (Fig. 4B), mainly in the white matter of the left temporoparietal lobe. Diffusion-weighted imaging showed a small high intensity area of hematoma in the right convexity (Fig. 4D). None of the CBF maps in these 3 cases showed decreased CBF with laterality, whereas all the CBV maps showed increased or normal CBV levels in the area of increased MTT.

Intensive therapies were instituted as described in Methods, and the clinical vasospasm symptoms improved in all 3 patients. Similarly, $\mathrm{T}_{2}$-weighted MR imaging after treatment revealed that the diffusionperfusion mismatch area had recovered and was free from permanent cerebral infarction (Figs. 2F, $3 \mathrm{~F}$, and $4 \mathrm{~F}$ ). On the other hand, SPECT showed no changes in brain circulation at the same stage (Figs. $2 \mathrm{E}, 3 \mathrm{E}$, and $4 \mathrm{E}$ ).

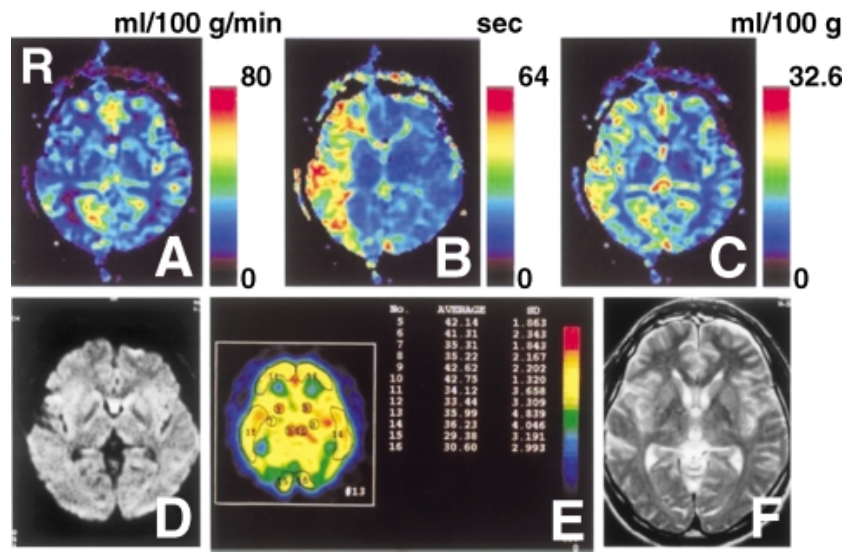

Fig. 2 Case 1 with diffusion-perfusion mismatch on Day 10. A-C: Perfusion-weighted magnetic resonance (MR) images showing normal cerebral blood flow (A), but increased mean transit time (MTT) (B) and cerebral blood volume (C) widely extending to the right hemisphere. D: Diffusion-weighted MR image showing no high intensity area except for very small high intensity signals of hematoma in the right sylvian fissure and the anterior horn of the lateral ventricle. E: Single photon emission computed tomography scan showing no apparent changes in brain circulation. $F: T_{2}$-weighted MR image on Day 17 showing no cerebral infarction in the area of increased MTT.

\section{Discussion}

The present study clearly demonstrated diffusionperfusion mismatch in 3 patients with vasospasm manifesting as somnolence, hemiparesis, or aphasia.

Theoretically, CBV increases at first in common ischemia with dilation of the brain vessels, before any decreases in CBF or increases in oxygen uptake ratio, as the partial cerebral circulation begins to decrease. ${ }^{22,23)}$ In this study, no decrease in CBF but a clear diffusion-perfusion mismatch was found at the apparent site of the clinical symptoms. This observation also shows that vasospasm already causes clinical symptoms with increased CBV due to hemodynamic compensation before any decrease in CBF occurs.

Perfusion-weighted imaging has been used to qualitatively assess MTT, CBV, and CBF in patients with vasospasm, and cerebral circulation was assessed only by relative comparison with the contralateral circulation or the surrounding circulation. ${ }^{24)}$ Another study of perfusion-weighted imaging with MTT, CBV, and TTP was likely to detect excessive increases in MTT or TTP, because the 
space resolution of perfusion-weighted imaging cannot measure changes of circulation in a very small area. ${ }^{13)}$

The present method could detect the diffusionperfusion mismatch clearly in a relatively large area matched to the clinical symptoms, based on quantitative CBF measurement by perfusion-weighted imaging which was confirmed by comparing with CBF measurements by SPECT on the same day. Our method could already clearly show the diffusion-

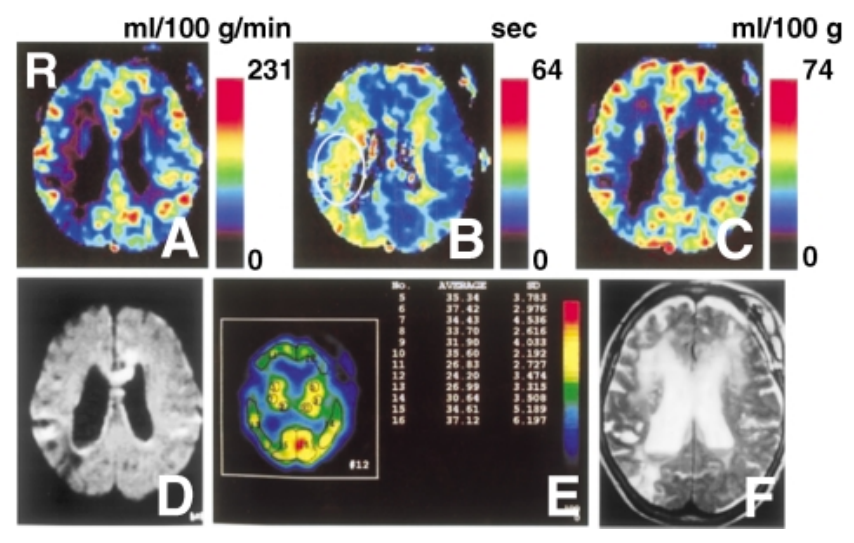

Fig. 3 Case 2 with diffusion-perfusion mismatch on Day 9. A-C: Perfusion-weighted magnetic resonance (MR) images showing normal cerebral blood flow (A), but increased mean transit time (MTT) (B) and cerebral blood volume (C) in the right frontal lobe (circle). D: Diffusion-weighted MR image showing no high intensity area except for some small high intensity signals of hematoma in the bilateral temporoparietal cerebral sulci and a small infarction in the white matter anterior to the left lateral ventricle. E: Single photon emission computed tomography scan showing no apparent changes in brain circulation. $\quad F: T_{2}$-weighted MR image on Day 54 showing no cerebral infarction in the area of increased MTT, except in an old right parietal infarction. perfusion mismatch before SPECT had revealed any abnormality.

SPECT provides direct information about tissue perfusion, but is not practical for the early detection of vasospasm. The detection of diffusion-perfusion mismatch is useful for identifying cerebral ischemia in patients with vasospasm in the early stage before SPECT and diffusion-weighted imaging can detect any abnormality. This method is anticipated to make a valuable contribution to the prevention of irreversible cerebral infarction in patients with vasospasm.

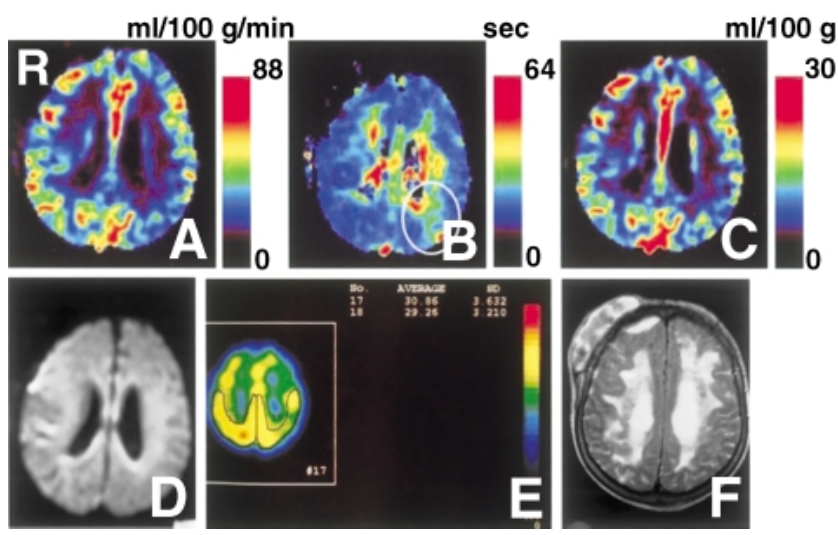

Fig. 4 Case 3 with diffusion-perfusion mismatch on Day 9. A-C: Perfusion-weighted magnetic resonance (MR) images showing normal cerebral blood flow (A) and cerebral blood volume (C), but increased mean transit time (MTT) (B), mainly in the white matter of the left temporoparietal lobe (circle). D: Diffusion-weighted MR image showing a small high intensity area of hematoma in the right convexity. E: Single photon emission computed tomography scan showing no apparent changes in brain circulation. $F$ : $\mathrm{T}_{2}$-weighted MR image on Day 33 showing no cerebral infarction in the area of increased MTT.

Table 1 Comparison of clinical deficits with magnetic resonance imaging and single photon emission computed tomography (SPECT) findings

\begin{tabular}{|c|c|c|c|c|c|c|c|c|}
\hline $\begin{array}{l}\text { Case } \\
\text { No. }\end{array}$ & $\begin{array}{l}\text { Age } \\
\text { (yrs)/ } \\
\text { Sex }\end{array}$ & $\begin{array}{l}\text { Hunt and } \\
\text { Kosnik } \\
\text { grade }\end{array}$ & $\begin{array}{l}\text { Days to } \\
\text { clipping } \\
\text { from onset }\end{array}$ & $\begin{array}{l}\text { Days to } \\
\text { examination } \\
\text { from onset }\end{array}$ & $\begin{array}{c}\text { Symptoms } \\
\text { at examination }\end{array}$ & $\begin{array}{l}\text { Low } \\
\text { perfusion } \\
\text { area on } \\
\text { SPECT }\end{array}$ & $\begin{array}{l}\text { Resultant } \\
\text { infarction }\end{array}$ & $\begin{array}{c}\text { Outcome } \\
\text { (modified } \\
\text { Rankin } \\
\text { scale) }\end{array}$ \\
\hline 1 & $39 / \mathrm{M}$ & 3 & 1 & 10 & $\begin{array}{l}\text { somnolence, } \\
\text { lt hemiparesis }\end{array}$ & no & - & 0 \\
\hline 2 & $79 / F$ & 3 & 1 & 9 & $\begin{array}{l}\text { somnolence, } \\
\text { lt hemiparesis }\end{array}$ & no & - & 4 \\
\hline
\end{tabular}




\section{Acknowledgments}

We thank Mr. Yoshiyuki Igarashi for his technical support and advice regarding SPECT and Mrs. Tomomi Ohtonari for her assistance in preparing the manuscript for submission.

\section{References}

1) Assalid R, Huber $P$, Nornes $H$ : Evaluation of cerebrovascular spasm with transcranial Doppler ultrasound. J Neurosurg 60: 37-41, 1984

2) Astrup J, Siesjo BK, Symon L: Thresholds in cerebral ischemia - the ischemic penumbra. Stroke 12: 723725, 1981

3) Ay H, Buonanno FS, Rordorf G, Schaefer PW, Schwamm LH, Wu O, Gonzalez RG, Yamada K, Sorensen GA, Koroshetz WJ: Normal diffusionweighted MRI during stroke-like deficits. Neurology 52: 1784-1792, 1999

4) Baird AE, Warach S: Magnetic resonance imaging of acute stroke. J Cereb Blood Flow Metab 18: 583-609, 1998

5) Bracard S, Anxionnat R, Auliac S, Melo Neto J, Lebendinsky A, Audibert G, Picard L: Relevance of diffusion and perfusion weighted mri for endovascular treatment of vasospasm in subarachnoid hemorrhage. J Neuroradiol 28: 27-32, 2001

6) Creissard P, Proust F: Vasospasm diagnosis: theoretical sensitivity of transcranial Doppler evaluated using 135 angiograms demonstrating vasospasm. Practical consequences. Acta Neurochir (Wien) 131: 1218, 1994

7) Donnan GA, Howells DW, Markus R, Toni D, Davis SM: Can the time window for administration of thrombolytics in stroke be increased? CNS Drugs 17: 1153-1159, 2005

8) Fukui MB, Johnson DW, Yonas H, Sekhar L, Latchaw RE, Pentheny S: Xe/CT cerebral blood flow evaluation of delayed symptomatic cerebral ischemia after subarachnoid hemorrhage. AJNR Am J Neuroradiol 13: 265-270, 1992

9) Harders AG, Gilsbach JM: Time course of blood velocity changes related to vasospasm in the circle of Willis measured by transcranial Doppler ultrasound. J Neurosurg 66: 718-728, 1987

10) Hatazawa J, Shimosegawa E, Toyoshima H, Ardekani BA, Suzuki A, Okudera T, Miura Y: Cerebral blood volume in acute brain infarction: A combined study with dynamic susceptibility contrast MRI and 99mTc-HMPAO-SPECT. Stroke 30: 800-806, 1999

11) Hennerici M, Rautenberg W, Schwartz A: Transcranial Doppler ultrasound for the assessment of intracranial arterial flow velocity - Part 2. Evaluation of intracranial arterial disease. Surg Neurol 27: 523-532, 1987

12) Heros RC, Zervas NT, Varsos V: Cerebral vasospasm after subarachnoid hemorrhage: an update. Ann Neurol 14: 599-608, 1983
13) Hertel F, Walter C, Bettag M, Mörsdorf M: Perfusionweighted magnetic resonance imaging in patients with vasospasm: a useful new tool in the management of patients with subarachnoid hemorrhage. Neurosurgery 56: 28-35, 2005

14) Kawamura S, Sayama I, Yasui N, Uemura K: Sequential changes in cerebral blood flow and metabolism in patients with subarachnoid haemorrhage. Acta Neurochir (Wien) 114: 12-15, 1992

15) Knuckey NW, Fox RA, Surveyor I, Stokes BA: Early cerebral blood flow and computerized tomography in predicting ischemia after cerebral aneurysm rupture. J Neurosurg 62: 850-855, 1985

16) Mani RL, Eisenberg RL: Complications of catheter cerebral arteriography: analysis of 5, 000 procedures. II. Relation of complication rates to clinical and arteriographic diagnoses. AJR Am J Roentgenol 131: 867-869, 1978

17) Mani RL, Eisenberg RL, McDonald EJ Jr, Pollock JA, Mani JR: Complications of catheter cerebral arteriography: analysis of 5, 000 procedures. I. Criteria and incidence. AJR Am J Roentgenol 131: 861-865, 1978

18) Mickey B, Vorstrup S, Voldby B, Lindewald H, Harmsen A, Lassen NA: Serial measurement of regional cerebral blood flow in patients with $\mathrm{SAH}$ using 133Xe inhalation and emission computerized tomography. J Neurosurg 60: 916-922, 1984

19) Moonis $\mathrm{M}$ : Imaging in acute ischemic stroke: relevance to management. Neurol India 50: S30-36, 2002

20) Ohkuma H, Suzuki S, Kubo K, Islam S, Kikkawa T: Cortical blood flow during cerebral vasospasm after aneurysmal subarachnoid hemorrhage: three-dimensional N-isopropyl-p-[123I]iodoamphetamine single photon emission CT findings. AJNR Am J Neuroradiol 24: 444-450, 2003

21) Ostergaard L, Sorensen AG, Kwong KK, Weisskoff RM, Gyldensted C, Rosen BR: High resolution measurement of cerebral blood flow using intravascular tracer bolus passages. Part II: Experimental comparison and preliminary results. Magn Reson Med 36: 726-736, 1996

22) Powers WJ, Grubb RL Jr, Baker RP, Mintun MA, Raichle ME: Regional cerebral blood flow and metabolism in reversible ischemia due to vasospasm. Determination by positron emission tomography. J Neurosurg 62: 539-546, 1985

23) Powers WJ, Press GA, Grubb RL Jr, Gado M, Raichle ME: The effect of hemodynamically significant carotid artery disease on the hemodynamic status of the cerebral circulation. Ann Intern Med 106: 27-34, 1987

24) Rordorf G, Koroshetz WJ, Copen WA, Gonzalez G, Yamada K, Schaefer PW, Schwamm LH, Ogilvy CS, Sorensen AG: Diffusion- and perfusion-weighted imaging in vasospasm after subarachnoid hemorrhage. Stroke 30: 599-605, 1999

25) Seiler RW, Grolimund P, Aaslid R, Huber P, Nornes 
$\mathrm{H}$ : Cerebral vasospasm evaluated by transcranial ultrasound correlated with clinical grade and CTvisualized subarachnoid hemorrhage. J Neurosurg 64: 594-600, 1986

26) Sitburana O, Koroshetz WJ: Magnetic resonance imaging: implication in acute ischemic stroke management. Curr Atheroscler Rep 7: 305-312, 2005

27) Sloan MA, Haley EC Jr, Kassell NF, Henry ML, Stewart SR, Beskin RR, Sevilla EA, Torner JC: Sensitivity and specificity of transcranial Doppler ultrasonography in the diagnosis of vasospasm following subarachnoid hemorrhage. Neurology 39: 1514-1518, 1989

28) Sorensen AG, Copen WA, Ostergaard L, Buonanno FS, Gonzalez RG, Rordorf G, Rosen BR, Schwamm LH, Weisskoff RM, Koroshetz WJ: Hyperacute stroke: simultaneous measurement of relative cerebral blood volume, relative cerebral blood flow, and mean tissue transit time. Radiology 210: 519-527, 1999

29) Uno M, Harada M, Yoneda K, Matsubara S, Satoh K, Nagahiro S: Can diffusion- and perfusion-weighted magnetic resonance imaging evaluate the efficacy of acute thrombolysis in patients with internal carotid artery or middle cerebral artery occlusion? Neurosurgery 50: 28-35, 2002

30) Wechsler LR, Ropper AH, Kistler JP: Transcranial Doppler in cerebrovascular disease. Stroke 17: 905912, 1986

Address reprint requests to: Tatsuya Ohtonari, M.D., Department of Neurosurgery, Brain Attack Center Ota Memorial Hospital, 3-6-28 Okinogami-cho, Fukuyama, Hiroshima 720-0825, Japan.

e-mail: otonari1@alpha.ocn.ne.jp

\section{Commentary}

The authors describe a study in which diffusionweighted and perfusion-weighted magnetic resonance imaging (MRI) were investigated in an effort to detect diffusion-perfusion mismatch in the early stages of cerebral vasospasm. In this small series, 17 patients with aneurysmal subarachnoid hemorrhage were evaluated at an average of 7.2 days following hemorrhage with perfusion-weighted MRI and single photon emission computed tomography (SPECT). Of the 17 patients, 14 were asymptomatic and three had developed somnolence, hemiparesis or aphasia. No evidence of diffusion-perfusion mismatch was identified in the asymptomatic patients, whereas the three patients who experienced clinical deterioration were demonstrated to have increased mean transit time, normal cerebral blood flow and increased or normal cerebral blood volume. Diffusion-perfusion mismatch was clearly demonstrated in these patients.

This demonstration by the authors illustrates the potential for diffusion-weighted and perfusion-weighted MRI to contribute to the early detection of vasospasm and potentially reduce the incidence of permanent ischemic injury.

The primary criticisms of the paper are the small number of patients involved, particularly the small number of symptomatic patients. Furthermore, these patients had already developed clear evidence of neurological deficits that strongly suggested clinically significant vasospasm. Time required to perform the MRI studies as well as the SPECT studies could potentially delay the institution of early angiography with the potential for therapeutic angioplasty.

The authors are to be congratulated for their contribution and this study should stimulate a further investigation into the use of MRI in detecting patients at high risk of vasospasm before they clinically deteriorate.

$$
\begin{array}{r}
\text { Daniel L. BARRow, M.D. } \\
\text { Department of Neurosurgery } \\
\text { Emory University } \\
\text { Atlanta, Georgia, U.S.A. }
\end{array}
$$

Dr. Ohtonari et al. reported a selected group of 17 patients with aneurysmal subarachnoid hemorrhage (SAH). Three of them presented with symptomatic vasospasm (VSP) manifestations, which were confirmed by diffusion-perfusion mismatch, that meant no related diffusion-weighted $(\mathrm{d} w)$ MRI changes, cerebral blood flow (CBF) change in perfusion-weighted $(p w)$ MRI in all 3 cases, but increased mean transit time (MTT) and cerebral blood volume (CBV) in 2 cases, and increased MTT only in 1 case. After intensive therapy, 3 patients completely improved and T2weighted MRI and SPECT demonstrated no apparent abnormality (It is a pity there was no repeated pwMRI!)

It is a novel concept that increased MTT with or without increased CBV in pwMRI can predict the very early stage of symptomatic VSP, although the prediction value of diffusion-perfusion mismatch in VSP after $\mathrm{SAH}$ has been reported in the literature. However, caution should be exercised with MRI measurement of tissue blood flow with intravenous tracer. In addition, because the study is a retrospective nonrandomized analysis and authors investigated predominantly patients suspected to have VSP, there was a negative selection bias in the study. A prospective trial, therefore, is desirable to confirm the diagnostic and therapeutic relevance of increased MTT in pwMRI.

With regard to daily clinical practice, I prefer perfusion CT (pCT) to pwMRI, because pCT can be performed on a routine, easy and quick basis, and because most patients with suspected VSP are critical, with a tracheal tube that has to be changed to a nonmagnetic one.

Liang-Fu ZHOU, M.D. Department of Neurosurgery HuaShan Hospital, S.M.C., F.D.U. Shanghai, P.R.C. 